QUARTERLY OF APPLIED MATHEMATICS

VOLUME LXV, NUMBER 3

SEPTEMBER 2007, PAGES 523-528

$\mathrm{S} 0033-569 \mathrm{X}(07) 01058-1$

Article electronically published on June 6, 2007

\title{
EXISTENCE OF TURNING POINTS FOR THE RESPONSE DIAGRAM OF THE POISEUILLE FLOW WITH PRESCRIBED FLOW-RATE
}

\author{
BY \\ GIOVANNI CIMATTI \\ Dipartimento di Matematica, Largo Bruno Pontecorvo 5, Pisa, Italy
}

\begin{abstract}
We study the stationary Poiseuille flow in a cylindrical channel of arbitrary cross-section with temperature dependent viscosity and internal dissipation. We assume the flow-rate $\Phi$ given and the axial pressure gradient $\mu$ unknown. This leads to a nonlocal problem. We show the existence in the response diagram, the plane $(\Phi, \mu)$, of two turning points.
\end{abstract}

1. In this paper we study the stationary Poiseuille flow in a pipe, assuming the viscosity $\eta$ and the thermal conductivity $\kappa$ to be given functions of the temperature $u$. Let $\Omega$ be an open and bounded subset of $\mathbf{R}^{2}$ with a regular boundary $\Gamma$ representing the section of the pipe. If the axial pressure gradient $\mu$ is a given constant for the determination of the z-component $v(x, y)$ of the velocity and of the temperature $u$, we have (see [4]) the following Problem A: find $v(x, y)$ and $u(x, y)$ such that

$$
\begin{gathered}
-\nabla \cdot(\eta(u) \nabla v)=\mu \text { in } \Omega, v=0 \text { on } \Gamma, \\
-\nabla \cdot(\kappa(u) \nabla u)=\eta(u)|\nabla v|^{2} \text { in } \Omega, u=0 \text { on } \Gamma .
\end{gathered}
$$

The Navier-Stokes system, for the problem at hand, reduces to (1.1), whereas (1.2) is the energy equation. In the right hand side of equation (1.2) we have the heating source corresponding to the viscous forces. We suppose, on physical grounds,

$$
\eta(u) \in C^{4}\left(\mathbf{R}^{1}\right), \kappa(u) \in C^{4}\left(\mathbf{R}^{1}\right), \eta(u)>0, \kappa(u)>0 \text { for all } u \in \mathbf{R}^{1} .
$$

If $\mu=0$ it is easily seen that the only solution of Problem A is $v(x, y)=0, u(x, y)=0$ (see Lemma 1.2). Moreover, if $|\mu|$ is sufficiently small a branch of small solutions

Received September 28, 2006.

2000 Mathematics Subject Classification. Primary 76D03, 76D05.

Key words and phrases. Poiseuille flow, temperature dependent viscosity, existence and uniqueness of solutions.

E-mail address: cimatti@dm.unipi.it

(C)2007 Brown University Reverts to public domain 28 years from publication 
(functions of $\mu$ ) starts from the trivial solution $v=0, u=0$. This is proved in the following

Theorem 1.1. Let (1.3) hold; then there exists $\mu_{0}>0$ such that, if $|\mu|<\mu_{0}$, Problem A has one and only one solution, $(v(\mu), u(\mu)) \in C^{4}\left(\left(-\mu_{0}, \mu_{0}\right) ; C^{2, \alpha}(\bar{\Omega}) \times C^{2, \alpha}(\bar{\Omega})\right)$.

Proof. We apply the implicit function theorem in Banach spaces. Let

$$
X=\left\{u \in C^{2, \alpha}(\bar{\Omega}), u=0 \text { on } \Gamma\right\}, Y=C^{0, \alpha}(\bar{\Omega}) .
$$

Define $B=X \times X, C=Y \times Y$ and the map $\mathcal{G}: B \times \mathbf{R}^{1} \longrightarrow C$ given by

$$
\mathcal{G}(\mathbf{w}, \mu)=\left(\nabla \cdot(\eta(u) \nabla v)+\mu, \nabla \cdot(\kappa(u) \nabla u)+\eta(u)|\nabla v|^{2}\right), \mathbf{w}=(v, u) .
$$

We have $\mathcal{G}(\mathbf{0}, 0)=\mathbf{0}$ and $\mathcal{G} \in C^{4}\left(B \times \mathbf{R}^{1} ; C\right)$. The partial derivative of $\mathcal{G}$ with respect to $\mathbf{w}, \mathcal{G}_{\mathbf{w}}(\mathbf{w}, \mu)[\mathbf{W}]: B \times \mathbf{R}^{1} \longrightarrow \mathcal{L}\left(B \times \mathbf{R}^{1} ; C\right), \mathbf{W}=(V, U)$, is easily computed, and we find:

$$
\mathcal{G}_{\mathbf{w}}(\mathbf{0}, 0)[\mathbf{W}]=(\eta(0) \Delta V, \kappa(0) \Delta U) .
$$

By the properties of the Laplace operator, $\mathcal{G}_{\mathbf{w}}(\mathbf{0}, 0)$ is invertible with a bounded inverse. Hence there exists $\mu_{0}>0$ such that $\mathcal{G}(\mathbf{w}, \mu)=0$ is locally solvable with respect to $\mathbf{w}$ if $|\mu|<\mu_{0}$ and the solution $\mathbf{w}=\hat{\mathbf{w}}(\mu)$ belongs to $C^{4}\left(\left(-\mu_{0}, \mu_{0}\right) ; B\right)$.

We have

Lemma 1.2. Let $\hat{\mathbf{w}}(\mu)=(v(x, y ; \mu), u(x, y ; \mu))$ be the solution to Problem A given by Theorem 1.1 when $|\mu|<\mu_{0}$. Then

$$
\begin{gathered}
v(x, y ; 0)=0, \\
u(x, y ; 0)=0, \\
v(x, y ; \mu)=-v(x, y ;-\mu), \\
u(x, y ; \mu)=u(x, y ;-\mu), \\
w_{\mu \mu}(x, y ; 0)=0 \text { in } \bar{\Omega}, \\
u_{\mu}(x, y ; 0)=0 \text { in } \bar{\Omega} .
\end{gathered}
$$

Proof. If $\mu=0$, after multiplication by $v$ and integration by parts over $\Omega$, we get from (1.3)

$$
\int_{\Omega} \eta(u)|\nabla v|^{2} d x d y=0
$$

thus we have (1.4). Equation (1.5) follows in a similar way from (1.4). Define $\hat{v}(x, y ; \mu)=$ $-v(x, y ;-\mu)$ and $\hat{u}(x, y ; \mu)=u(x, y ;-\mu)$. It is easy to see that $\hat{v}, \hat{u}$ satisfies (1.1), (1.2). Since we have $|\mu|<\mu_{0}$ and therefore Problem A has one and only one solution, we conclude that (1.6), (1.7) hold. Equations (1.8) and (1.9) are immediate consequences of the regularity with respect to $\mu$ and of (1.6) and (1.7).

Remark 1.3. For a proof of existence, but not of uniqueness for Problem A, we refer to [4].

In a different, but equally legitimate formulation of the Poiseuille flow (see [1] and 2]) we may consider the axial pressure gradient $\mu$ as an unknown constant and assume the 
flow rate

$$
\Phi=\int_{\Omega} v d x d y
$$

to be given. In this way we have the following non-local Problem B: find $v(x, y), u(x, y)$ and $\mu \in \mathbf{R}^{1}$ such that

$$
\begin{gathered}
-\nabla \cdot(\eta(u) \nabla v)=\mu \text { in } \Omega, v=0 \text { on } \Gamma, \\
-\nabla \cdot(\eta(u) \nabla u)=\eta(u)|\nabla v|^{2} \text { in } \Omega, u=0 \text { on } \Gamma, \\
\Phi=\int_{\Omega} v d x d y .
\end{gathered}
$$

If $v(x, y ; \mu), u(x, y ; \mu)$ is a given solution to Problem $\mathrm{A}$ and we define the function

$$
\varphi(\mu)=\int_{\Omega} v(x, y ; \mu) d x d y,
$$

the local solutions of Problem B in $|\mu|<\mu_{0}$ are in a one-to-one correspondence with the solutions of the equation

$$
\varphi(\mu)=\Phi
$$

Aim of this note is to prove that, under suitable hypotheses, the response diagram in the plane $(\Phi, \mu)$ has two turning points (see figure below).

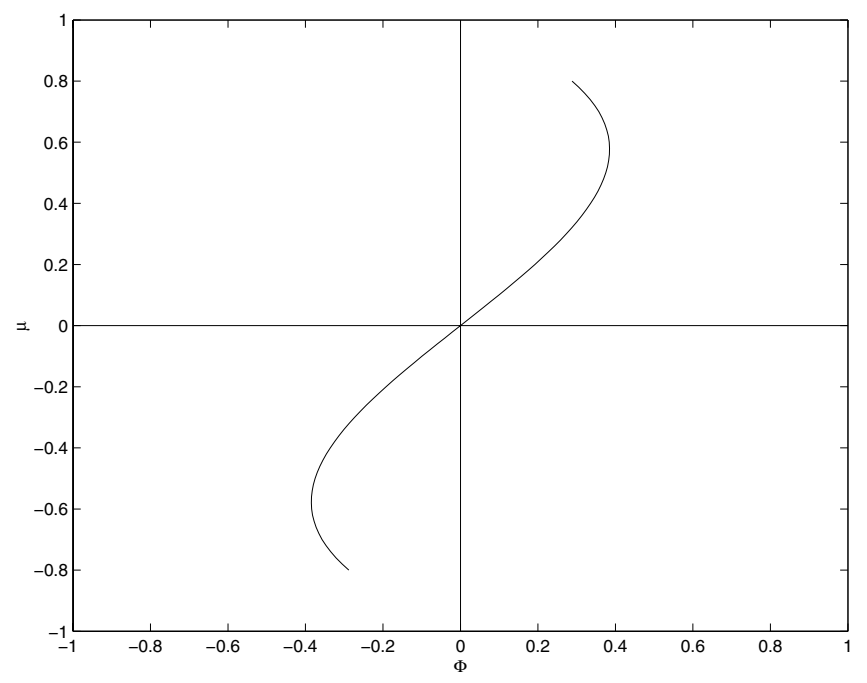

FIG. 1.

Thus two different triplets $\left(v_{1}, u_{1}, \mu_{1}\right),\left(v_{2}, u_{2}, \mu_{2}\right)$ may correspond to the same flow rate $\Phi$. From (1.6) we can say that $\varphi(\mu)$ is an odd function of class $C^{4}(-\mu, \mu)$. Therefore

$$
\begin{gathered}
\varphi(0)=0, \\
\varphi^{\prime \prime}(0)=0 .
\end{gathered}
$$


To make a local study of $\varphi(\mu)$ near $\mu=0$ we also need information about $\varphi(0)$ and $\varphi^{\prime \prime \prime}(0)$. To this end, we note that $v_{\mu}(x, y ; 0)$ is given by the solution of the problem

$$
-\nabla \cdot\left(\eta(0) \nabla v_{\mu}(x, y ; 0)\right)=1 \text { in } \Omega, v_{\mu}(x, y ; 0)=0 \text { on } \Gamma .
$$

By the maximum principle we have, in view of (1.3),

$$
v_{\mu}(x, y ; 0)>0 \text { in } \bar{\Omega} \text {. }
$$

This implies

$$
\varphi^{\prime}(0)>0
$$

and the following

Lemma 1.4. There exist two constants $\Phi_{1}>0$ and $\mu_{1}>0$ such that, if $|\Phi|<\Phi_{1}$, Problem B has one and only one solution,

$$
(v(x, y ; \mu), u(x, y ; \mu), \mu) \in C^{2, \alpha}(\bar{\Omega}) \times C^{2, \alpha}(\bar{\Omega}) \times\left(-\mu_{1}, \mu_{1}\right) .
$$

To compute $\mu^{\prime \prime \prime}(0)$ we first need $u_{\mu \mu}(x, y ; 0)$, which is easily found to satisfy the problem:

$$
-\nabla \cdot\left(\kappa(0) \nabla u_{\mu \mu}(x, y ; 0)\right)=2 \eta(0)\left|\nabla v_{\mu}(x, y ; 0)\right|^{2} \text { in } \Omega, u_{\mu \mu}(x, y ; 0)=0 \text { on } \Gamma \text {. }
$$

By the maximum principle we have:

$$
u_{\mu \mu}(x, y ; 0)>0 \text { in } \bar{\Omega} .
$$

Once $u_{\mu \mu}(x, y ; 0)$ is known, we obtain $v_{\mu \mu \mu}(x, y ; 0)$ from

$$
\begin{gathered}
-\nabla \cdot\left(\eta(0) \nabla v_{\mu \mu \mu}(x, y ; 0)\right)=3 \eta^{\prime}(0)\left[\nabla u_{\mu \mu}(x, y ; 0) \cdot \nabla v_{\mu}(x, y ; 0)-\frac{1}{\eta(0)} u_{\mu \mu}(x, y ; 0)\right] \begin{array}{l}
\text { in } \Omega, \\
\left.v_{\mu \mu \mu}(x, y ; 0)=0 \text { on } \Gamma .22\right)
\end{array}
\end{gathered}
$$

We have

$$
\varphi^{\prime \prime \prime}(0)=\int_{\Omega} v_{\mu \mu \mu}(x, y ; 0) d x d y .
$$

By (1.18) $\varphi(0)$ is always positive; on the other hand $\varphi^{\prime \prime \prime}(0)$ may be either positive or negative (see example below). In this second case we have the following

Theorem 1.5. Let $(v(x, y ; \mu), u(x, y ; \mu))$ be the solution to Problem A given by Theorem 1.1 if $|\mu|<\mu_{0}$ and

$$
\varphi(\mu)=\int_{\Omega} v(x, y ; \mu) d x d y .
$$

Assume

$$
\varphi^{\prime \prime \prime}(0)<0 .
$$

Then a constant $a>0$ exists such that if

$$
\varphi^{\prime}(0)<a
$$

there are four positive numbers $\Phi_{1}, \Phi_{2}, \delta_{1} \delta_{2}$ such that, when

$$
\Phi_{1}<\Phi<\Phi_{2},
$$


Problem B has exactly two solutions:

$$
\left(v_{1}\left(x, y ; \mu_{1}\right), u_{1}\left(x, y ; \mu_{1}\right), \mu_{1}\right),\left(v_{2}\left(x, y ; \mu_{2}\right), u_{2}\left(x, y ; \mu_{2}\right), \mu_{2}\right)
$$

with $\mu_{1}, \mu_{2} \in\left(\delta_{1}, \delta_{2}\right)$.

Proof. Define

$$
g(\mu)=\varphi^{\prime}(0)-\varphi(\mu) .
$$

We have $g(\mu) \in C^{2}\left(-\mu_{0}, \mu_{0}\right)$. Moreover, by (1.16) and (1.25),

$$
\begin{gathered}
g^{\prime}(0)=-\varphi^{\prime \prime}(0)=0, \\
g^{\prime \prime}(0)=-\varphi^{\prime \prime \prime}(0)>0 .
\end{gathered}
$$

Hence there exists $a>0$ and $\bar{\mu}>0$ such that, if (1.26) holds, the equation

$$
g(\mu)=\varphi^{\prime}(0)
$$

has in $(0, \hat{\mu})$ one and only one solution $\mu^{*}$ i.e.

$$
g\left(\mu^{*}\right)=\varphi^{\prime}(0)
$$

and

$$
\begin{gathered}
g^{\prime}(\mu)<0 \text { in }(-\bar{\mu}, 0), \\
g^{\prime}(\mu)>0 \text { in }(0, \bar{\mu}) .
\end{gathered}
$$

On the other hand, (1.31) is equivalent to

$$
\varphi^{\prime}\left(\mu^{*}\right)=0
$$

and by (1.33) we have

$$
\varphi^{\prime \prime}(\mu)=-g^{\prime}(\mu)<0 \text { in }(0, \bar{\mu}) .
$$

Therefore, four numbers exist: $\mu_{1}>0, \mu_{2}>0, \Phi_{1}>0, \Phi_{2}>0$ with

$$
0<\mu_{1}<\mu^{*}<\mu_{2}
$$

such that, if (1.27) holds, the equation

$$
\varphi(\mu)=\Phi
$$

has in the interval $\left(\delta_{1}, \delta_{2}\right)$ exactly two solutions, $\mu_{1}, \mu_{2}$. By Theorem 1.1, Problem A has one and only one solution for each of the values $\mu_{1}$ and $\mu_{2}$. Thus we obtain two different solutions of Problem B corresponding to the same value of $\Phi$.

Remark 1.6. The constant $a$ entering in Theorem 1.5 depends only on $\varphi^{\prime \prime}(0)$. Therefore condition (1.26) is meaningful.

Crucial in Theorem 1.5 is condition (1.25). To prove that there are situations in which (1.25) is verified we consider the special, but important case of a pipe of circular crosssection of radius $R$. Assuming $\mu$ to be in the range of uniqueness for Problem A, $v$ and 
$u$ depend only on $r=\sqrt{x^{2}+y^{2}}$. It is easily found that

$$
\begin{gathered}
v_{\mu}(r ; 0)=\frac{1}{4 \eta(0)}\left(R^{2}-r^{2}\right), \\
\varphi^{\prime}(0)=\frac{1}{8 \eta(0)} \pi R^{4}, \\
v_{\mu \mu \mu}(r ; 0)=-\frac{\eta^{\prime}(0)}{128 \eta^{3}(0) \kappa(0)}\left[r^{2}\left(r^{4}-3 R^{4}\right)+2 R^{6}\right], \\
\varphi^{\prime \prime \prime}(0)=-\frac{2 \pi \eta^{\prime}(0) R^{8}}{512 \eta^{3}(0) \kappa(0)} .
\end{gathered}
$$

Thus the sign of $\varphi^{\prime \prime \prime}(0)$ depends only on $\eta^{\prime}(0)$. For the absolute majority of fluids we have $\eta^{\prime}(0)<0$, however there are special substances (work-hardening fluids) for which $\eta^{\prime}(0)>0$ holds.

\section{REFERENCES}

[1] H. Beirão Da Veiga, Time-Periodic Solutions of the Navier-Stokes Equations inUnbounded Cylindrical Domains - Leray's Problem for Periodic Flow, Arch. Rational Mech. Anal. 178 (2005) 301-325. MR2196495 (2006k:35209)

[2] G. P. Galdi and A. M. Robertson, The Relation Between Flow Rate and Axial Pressure Gradient for Time-Periodic Poiseuille Flow in a Pipe, J. Math. Fluid. Mech. 7 (2005) 215-223. MR2192849 (2006j:76038)

[3] G. P. Galdi, An Introduction to the Mathematical Theory of the Navier-Stokes Equations: Linearized Steady Problems, Revised Edition, Springer Tracts in Natural Philosophy, Vol. 38, SpringerVerlag, New York, 1998. MR 1284205(95i:35216a)

[4] G. Cimatti, The Poiseuille Solution with Temperature Dependent Viscosity, Applicable Analysis, Vol. 84 (2005) 451-461. MR2151274 (2006a:35247) 